\title{
A Comparative Study of Correspondence-Search Algorithms in MIS Images
}

\author{
Gustavo A. Puerto and Gian-Luca Mariottini \\ Department of Computer Science and Engineering, \\ University of Texas at Arlington, 416 Yates Street, 76019 Texas
}

\begin{abstract}
The ability to find image similarities (feature matching) between laparoscopic views is essential in many robotic-assisted MinimallyInvasive Surgery (MIS) applications. Differently from feature tracking methods, feature matching does not make any restrictive assumption about the sequential nature of the two images or about the organ motion, and could then be used, e.g., to recover tracked features that were lost due to a prolonged occlusion, a sudden endoscopic-camera retraction, or a strong illumination change. This paper provides researchers in the medical-imaging computing community with an extensive comparison of the most up-to-date feature-matching algorithms over a large (and annotated) data set of 100 MIS-image pairs obtained from real interventions. The accuracy of these methods, as well as their ability to consistently retrieve as many good matches as possible, are evaluated for popular feature detectors. In addition, the dataset and the software implementations of these methods are made freely available on the Internet.
\end{abstract}

\section{Introduction}

In robotic-assisted Minimally-Invasive Surgery (MIS), the ability to find image similarities (feature matching) between laparoscopic views of the same scene is an essential need in many applications, such as shape recovery [1],2, calibration [3], structure and camera-motion estimation [4, 5, augmented reality [6, 7, etc.

Thus far, some algorithms have addressed the similarity-search problem in MIS video sequences only in the case that the two views are consecutive frames of a vided 1 , or when the organ motion is periodic 8,9 .

On the contrary, feature-matching algorithms [10 13] make very limited (or no) assumptions about the observed scene, or about the two given images. These algorithms have thus the potential to become a core-component in each of the aforementioned MIS applications, as they could be used to recover tracked features lost due to a prolonged occlusion, a sudden endoscopic-camera retraction, or a strong illumination change.

It is then of primary importance to provide researchers in the medical-imaging computing community with a comparative study of the accuracy of each of the aforementioned algorithms, and to evaluate their ability to consistently retrieve

1 This problem is usually known as feature tracking. 
as many good matches as possible. Existing works in the computer-vision field have thus far examined only the performance of feature detection 14 16, while other works have compared matching strategies in MIS [17, 18, based only on local appearance of descriptors, which are not robust to ambiguities.

In this work we present an extensive comparison of the aforementioned stateof-the-art feature-matching techniques [10 13. over a large (and annotated) data set of 100 MIS-image pairs obtained from real interventions. In particular, we measure both the ability of the methods to establish correct correspondences, as well as their accuracy in a tracking-recovery scenario. To the best of our knowledge, this is the first work that thoroughly compares and analyzes featurematching algorithms; in addition, we make available on the Internet the dataset and the software implementations of these methods 2

The paper is organized as follows: Sect. 2 provides a brief overview and discussion of the above feature-matching algorithms. Sect. 3 illustrates the results of our extensive experimental evaluation and discusses the algorithms' performance. Finally, Sect. 4 draws conclusions and illustrates future extensions.

\section{Overview of Feature-Matching Algorithms}

We consider a pair of images, $\mathcal{I}^{t}$ (training, e.g., before occlusion) and $\mathcal{I}^{q}$ (query, e.g., after occlusion), and two corresponding sets of image features (e.g., SIFT [10], SURF [14, ASIFT [19], etc.) $\mathcal{F}^{t}$ and $\mathcal{F}^{q}$, extracted from $\mathcal{I}^{t}$ and $\mathcal{I}^{q}$, respectively. Each image feature consists of a keypoint [10] (with pixel position, $\mathbf{x} \triangleq[x, y]^{T}$, scale $\sigma$, and orientation $\theta$ ), and of a descriptor vector, which captures the local appearance around the keypoint position.

The main goal of a feature-matching algorithm is to retrieve pairs of similar features (correspondences) among two images. Existing strategies, first find a set of initial (or candidate) matches, $\mathcal{M}$, by using appearance-based criteria that relies on the similarity between descriptors [15, 17. Among the most common criteria: simple thresholding, Nearest Neighbor (NN) distance ratio, etc.

Existing feature-matching algorithms mostly differ for their geometric-constraint phase, which is adopted to reduce the number of ambiguous matches originated by image regions with similar appearance.

Lowe's [10]: This method estimates a single Affine Transformation (AT) 20], A, which maps keypoints, $\mathbf{x}^{t}$ and $\mathbf{x}^{q}$, from $\mathcal{I}^{t}$ to $\mathcal{I}^{q}$.

Among the initial matches, $\mathcal{M}$, only those that obey to such a geometric AT will be considered as inliers, $\mathcal{M}_{i n}$. In particular, these inliers consist of those matches with a low symmetric keypoint reprojection error, $e_{s}$. For a pair of generic points, $\mathbf{u}^{t} \in \mathcal{I}^{t}$ and $\mathbf{u}^{q} \in \mathcal{I}^{q}, e_{s}$ is defined as follow 3 ,

$$
e_{s}\left(\mathbf{u}^{t}, \mathbf{u}^{q}, \mathbf{A}\right)=0.5\left\|\tilde{\mathbf{u}}^{q}-\mathbf{A} \tilde{\mathbf{u}}^{t}\right\|_{2}+0.5\left\|\mathbf{A}^{-1} \tilde{\mathbf{u}}^{q}-\tilde{\mathbf{u}}^{t}\right\|_{2}
$$

\footnotetext{
${ }^{2}$ Web: http://ranger.uta.edu/\% $\%$ egianluca/feature $\% 5$ fmatching.

${ }^{3} \tilde{\mathbf{u}}$ represents the extension to homogeneous coordinates of $\mathbf{u}$.
} 
Discussion: The approach in [10] works well for matching features on planar objects. However, since the majority of features in a MIS scenario lie on nonplanar (or deformable) surfaces, this method can only recover few matches. For example, from our experimental results, we observed that the refined matches (inliers) that better support $\mathbf{A}$ will only be those at the intersection of the object surface and of its (single) tangent plane. The other matches, distributed over the rest of the surface, will be considered as outliers.

Adaptive Multi-Affine (AMA) [11]: $\quad$ AMA relaxes the assumption of [10] by estimating a set of multiple ATs. Each AT is associated to a cluster of matches; as a result, the inliers (matches) detected by AMA will be distributed along the entire organ's surface. In AMA a set of clusters are first estimated as in [10, which are then sent to a cascade of RANSAC-based affine estimators. For each cluster, this estimation phase computes those features supporting a single AT $\mathbf{A}_{i}$. These clusters are then adaptively quantized by $k$-means, and each transformation is estimated, together with their inliers $\mathcal{M}_{i}$.

Discussion: AMA extracts more inliers than Lowe's approach. The final inliers in the image are spatially distributed over the entire organ's surface.

Agglomerative Correspondence Clustering (ACC) [12: ACC determines the set of refined matches by employing a hierarchical clustering algorithm based on an agglomerative (bottom-up) strategy. This strategy iteratively merges pairs of matches (or clusters of matches) into a single cluster based on a dissimilarity measure between matches (or clusters). This dissimilarity measure consists of both geometric and appearance constraints. Finally, the algorithm iteratively merges clusters according to both their dissimilarity measure and a linkage criteria, to generate the final clusters.

Discussion: ACC automatically detects similar portions in the two images without requiring previous knowledge of the scene. However, ACC requires the user to specify a larger number of (non-intuitive) parameters than [10,11.

Del Bimbo's Local Homography Mapping (DBA) [13]: DBA generates a set of $n$ synthetic images, $\mathcal{I}_{i}^{t}, i=1, \ldots, n$, obtained by applying to $\mathcal{I}^{t}$ a set of known random homographies $\mathbf{H}_{i}^{t}$ [20]. From the set, $\mathcal{F}^{\prime}$, of all the extracted features, a geometric constraint is used to remove repeated ones. The homography $\mathbf{H}_{i}$ and the associated inliers are then estimated by weighting each synthetic homography according to the remaining features. A final appearancebased phase (e.g., NCC) is used to removes the remaining outliers.

Discussion: DBA requires a large number of random (thus uncontrolled) synthetic images. As a result, DBA can only detect a low number of matches.

\section{Experimental Results and Discussion}

We compared the performances of the above algorithms (Sect. 2), in two scenarios: i) a highly controlled in-lab test with a non-planar object, and ii) a large 
MIS image dataset (100 image pairs) acquired from six surgical videos (with many cases of occlusions, large camera motion, specular reflections, etc.)

In each scenario, the performances of each algorithm have been assessed by measuring both the efficiency in detecting correct matches (matching performance), and the accuracy in mapping corresponding points between image pairs (mapping performance). For each image pair, a set of manually-labelled matches has been used to evaluate the matching performance by comparing the Receiver Operating Characteristic (ROC) curves [21], which depict the relative trade-off between the sensitivity (algorithm's capability to successfully retrieve correct matches, i.e., benefits), and the 1-specificity (i.e., algorithm's proficiency to erroneously retrieve wrong matches, i.e, costs). The mapping performance is measured by using a set of manually-labelled (ground-truth) corresponding points, and by measuring statistics on the symmetric reprojection error [20].

Similarly to [12,15, 17, 18, our database consists of a set of manually-labelled (ground-truth) SIFT matches captured by an expert user. Each match was manually labeled as 'correct' or 'wrong' by carefully observing their position on the two images (note that only the most certain matches were labelled as 'correct'). In addition, our database contains a set of manually-selected correspondences between each image pair. Our database also includes sets of initial SURF and ASIFT matches, however they are not labeled due to the large number of their initial matches and the associated complex labelling process 4 . Note that we have chosen to avoid to limit our datasets only to the strongest SURF and ASIFT features in order not to alter the performance (e.g., the percentage of correct matches) of these feature detectors.

Note that, for a fair comparison, all the aforementioned algorithms use the same sets of initial matches obtained by using $\mathrm{NN}$ distance ratio with threshold values 5 in the range of 0.8 and 0.9 . The parameters and thresholds of each algorithm were chosen to maximize efficiency. In particular, we used a threshold for the affine model of 5 pixels for both AMA and Lowe; a cutoff value of 5 was chosen for the ACC's linkage function, and a normalized cross correlation coefficient of 0.7 for DBA.

\subsection{In-Lab Experiments}

Two views of a non-planar object were used as a highly-controlled in-lab experiment (resolution $640 \times 480$ ). The initial 354 SIFT matches $(220$ were labelled as correct), while the SURF, and the ASIFT initial matches are 330, and 4903, respectively. In addition, 41 corresponding corners were manually selected.

Fig. 1 shows the ROC curves for each of the four algorithms parametrized by their score values. In Lowe and AMA the scores represent the negative

\footnotetext{
${ }^{4}$ For example, ASIFT can produce more than 4000 initial matches per image, thus rendering the ground-truth (manual) labelling almost unfeasible.

${ }^{5}$ These thresholds were chosen to maximize the number of initial matches. This choice does not affect our analysis since the initial matches are common to all the methods.

${ }^{6}$ Algorithm's quantitative measure for the classification task.
} 
symmetric-reprojection errors; for ACC the scores are the dissimilarity measure of the matches, which is also based on the symmetric reprojection error. For DBA, the scores represent the absolute value of the normalized cross-correlation coefficients. The square markers in Fig. 11(a), represent different score values, $\{5,20,50,70\}$ for Lowe, AMA, ACC, and $\{0.9,0.8,0.7,0.5\}$ for DBA. Fig. 1 (b) depicts a more focused comparison of the sensitivity values for Lowe, AMA and ACC7. The marked points have each an associated score value.

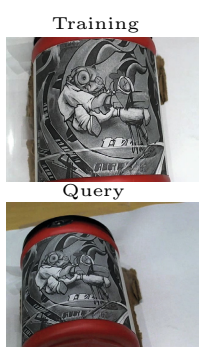

(a)

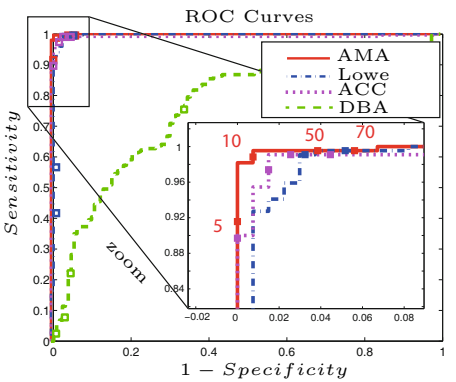

(b)

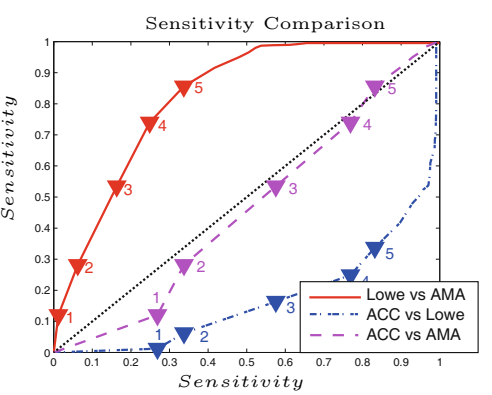

(c)

Fig. 1. In-lab experiment - Matching performance: (a) Query and Training images; (b) ROC curves of the four algorithms. AMA, ACC and Lowe are very competitive, being AMA slightly superior (c.f., inset); (b) Sensitivity comparison.

Table 1. In-lab experiment - Mapping performance

\begin{tabular}{|c||c|c|c||c|c|c||c|c|c|}
\hline Alg. & $\begin{array}{c}\text { Avg.Err. } \\
\text { SIft (pix) }\end{array}$ & $\begin{array}{c}\text { Time } \\
\text { SIFt }\end{array}$ & $\begin{array}{c}\text { Sen/1-Sp } \\
\text { SIFt }\end{array}$ & $\begin{array}{c}\text { Avg.Err. } \\
\text { surf (pix) }\end{array}$ & $\begin{array}{c}\text { Time } \\
\text { SURF }\end{array}$ & $\begin{array}{c}\text { \%Inl. } \\
\text { SURF }\end{array}$ & $\begin{array}{c}\text { Avg.Err. } \\
\text { ASIFt (pix) }\end{array}$ & $\begin{array}{c}\text { Time } \\
\text { ASIFt }\end{array}$ & $\begin{array}{c}\text { AnIfl. } \\
\text { ASIFt }\end{array}$ \\
\hline Lowe & $22.1 \pm 23.7$ & 0.22 & $0.28 / 0$ & $20.8 \pm 22.5$ & 0.16 & 17.3 & $19.3 \pm 21.0$ & 5.1 & 15.3 \\
\hline DBA & $32.9 \pm 64.7$ & 120 & $0.22 / 0.045$ & $17.4 \pm 27.2$ & 661 & 21.5 & $20.2 \pm 50.4$ & 721 & 25.9 \\
\hline AMA & $5.06 \pm 6.09$ & 1.26 & $0.98 / 0.001$ & $5.35 \pm 7.73$ & 1.48 & 60.0 & $2.62 \pm 2.81$ & 36.1 & 65.3 \\
\hline ACC & $6.33 \pm 8.9$ & 0.5 & $0.96 / 0.015$ & $7.46 \pm 8.13$ & 0.52 & 33.9 & $4.58 \pm 4.96$ & 433 & 42.3 \\
\hline
\end{tabular}

Table 1 provides statistical results for the mapping performance. The even columns show the mean and standard deviation of the symmetric reprojection error. The odd columns show the (SIFT) Sensitivity/1-specificity of Lowe, AMA, ACC (less than 5 pixels) and DBA (correlation coefficient greater than 0.7 ). For SURF and ASIFT, these columns contain the percentage of inliers with respect to the total number of matches, which indicate the detection power of each algorithm.

Discussion: The ROC curves in Fig. 1(a) show that Lowe, AMA and ACC, differently from DBA, have high matching performance. However, for the same threshold of 5 pixels, AMA has better performance than ACC and Lowe (c.f., Fig. 1(b)). An increase in the threshold value will provide an improved sensitivity for Lowe, AMA, and ACC, without compromising the 1-specificity.

\footnotetext{
${ }^{7}$ We did not include a comparison over 1-specificity because of space limits.
} 
The results in Table 1 show that AMA has superior mapping performances than the other methods, when using either SIFT, SURF, and ASIFT. This indicates that AMA (multiple ATs) can consistently detect as inliers many features lying on non-planar organs' surface. ACC achieves comparable results than AMA. However, AMA is computationally more expensive, than Lowe, and ACC (except when ASIFT features are used). Lowe (single AT) is the fastest method, but can only capture few matches. ACC exhibits a higher computational complexity when a large number of features is used (e.g., ASIFT), and DBA shows poor mapping performances caused by the random (thus uncontrolled generation of the synthetic images. We also observed that the mapping accuracy cannot be improved by simply increasing the AT-fitting thresholds for Lowe and AMA, since this could make the algorithms more sensitive to outliers.

\subsection{Surgical-Images Dataset}

Our MIS dataset consists of 100 images with a resolution of $704 \times 480$ pixels. The set of ground-truth correspondences has an average of 20 points. For SIFT features, the corresponding average ROC curves for each algorithm are illustrated in Fig. 2(a). The vertical lines indicate the confidence intervals of the mean with respect to different score values, with a significance level of 95\%. Fig. 2(b) shows the comparison of the sensitivity among pairs of (the most competitive) methods.

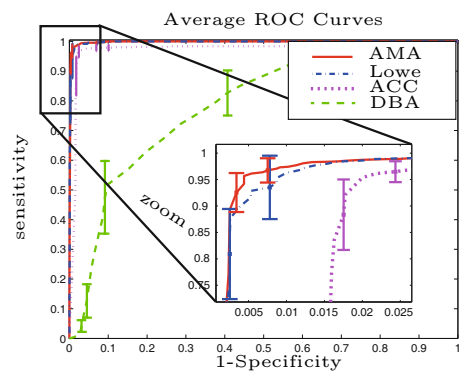

(a)

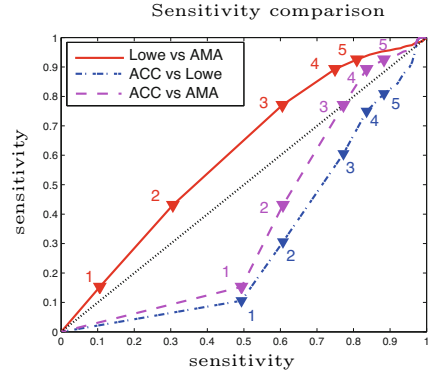

(b)

Fig. 2. MIS dataset - matching performance: (a) Average ROC curves. The vertical lines show the $95 \%$ confidence intervals. (b) Comparison of the algorithms' sensitivity.

Table 2 summarizes the average results for the algorithms' mapping performance for fixed threshold and for different types of features.

Discussion: AMA shows superiority in both metrics, followed by Lowe and ACC, with similar performances (see inset), while DBA is again far behind due to its incapability to filter out image similarities present in the MIS images. Observe that Lowe is significantly the fastest algorithm. Note that ACC's is faster than AMA and DBA for SIFT and SURF, while it becomes dramatically slower than

\footnotetext{
${ }^{8}$ Same scores values are used: $\{5,20,50,70\}$, and $\{0.9,0.8,0.7,0.5\}$.
} 
AMA with ASIFT. Differently from Lowe and ACC, AMA achieves good performances within the given threshold (5 pixels). AMA's best-mapping performance is attained when ASIFT features are used. We also observed that AMA cannot reliably detect isolated matches in the case of textureless regions.

ACC has similar sensitivity than AMA, but at a slightly larger 1-specificity. We observed that ACC dissimilarity function, which is not fully geometrical, is more flexible than Lowe's or AMA constraints. This flexibility allows ACC to capture matches even if they do not satisfy the affine map, but have similar appearance. However, this can produce an increased number of false positives and, thus, a larger mapping error. In addition, ACC has a large number of (nonintuitive) parameters to tune.

Table 2. MIS dataset: mapping performance

\begin{tabular}{|c|c|c|c|c|c|c|c|c|c|}
\hline Alg. & $\begin{array}{c}\text { Avg.Err. } \\
\text { SIFT }\end{array}$ & $\begin{array}{l}\text { Time } \\
\text { SIFT }\end{array}$ & \begin{tabular}{|c|} 
Sen/1-Sp. \\
SIFT
\end{tabular} & $\begin{array}{l}\text { Avg.Err. } \\
\text { SURF }\end{array}$ & $\begin{array}{l}\text { Time } \\
\text { SURF }\end{array}$ & $\begin{array}{l}\% \text { Inl. } \\
\text { SURF }\end{array}$ & \begin{tabular}{|c|} 
Avg.Err. \\
ASIFT
\end{tabular} & $\begin{array}{l}\text { Time } \\
\text { AsIft }\end{array}$ & \begin{tabular}{|l|}
$\%$ Inl. \\
ASIFT
\end{tabular} \\
\hline Lowe & $3.8 \pm 3.6$ & $0.3 \pm 0.1$ & $0.72 / .001$ & $3.6 \pm 2$ & $0.2 \pm 0.2$ & 39.6 & $3.6 \pm 3.4$ & $2.5 \pm 1.2$ & 42.3 \\
\hline DBA & $21 \pm 37$ & $122 \pm 14$ & $0.52 / .098$ & $23.3 \pm 30.3$ & $406 \pm 62$ & 25.1 & $21.6 \pm 30$ & $519 \pm 23$ & 38.3 \\
\hline AMA & $3.1 \pm 3.1$ & $1.0 \pm 0.6$ & \begin{tabular}{|l|}
$0.9 / .002$ \\
\end{tabular} & $3 \pm 3.1$ & $1.2 \pm 1.1$ & 59.4 & $3.3 \pm 3.1$ & $13.9 \pm 13.5$ & 54.5 \\
\hline $\mathrm{ACC}$ & $5.3 \pm 4.7$ & $0.3 \pm 0.1$ & $0.61 / .015$ & $7.1 \pm 8$ & $0.3 \pm 0.3$ & 59.0 & $10.4 \pm 13$ & $140 \pm 228$ & 53.5 \\
\hline
\end{tabular}

Fig. 3 shows an example of the matching performance of the four algorithms for given thresholds. Note that DBA has problems to filter out the ambiguities; Lowe and ACC obtain similar results, being AMA capable of retrieving a larger number of matches (covering most of the organ's surface).
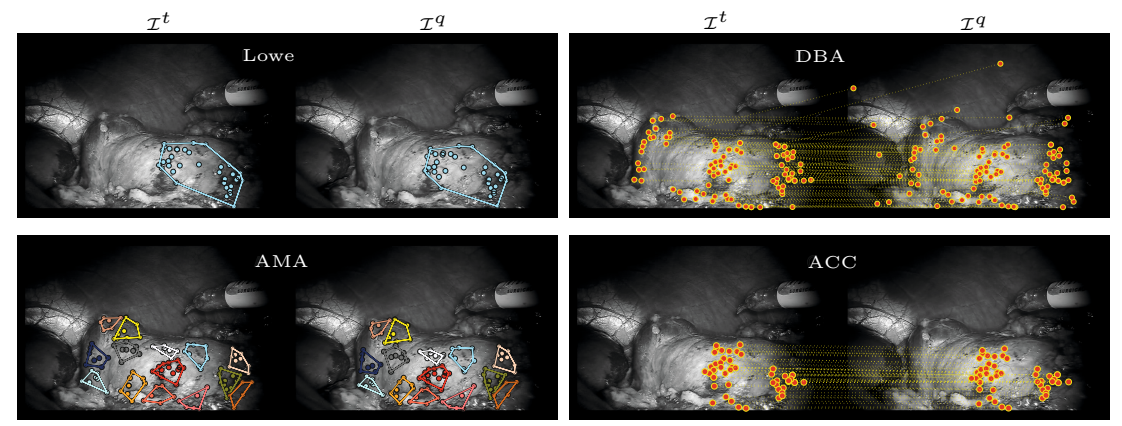

Fig. 3. Matching performances (better seen in color). Lowe only detects few matches limited to a portion of the organ. AMA, ACC and DBA retrieve matches distributed over all the organ. ACC and DBA are more sensitive to ambiguities.

\section{Conclusions}

This work presented for the first time a comparison of four state-of-the-art feature-matching algorithms. Differently from existing works, the four methods were compared among several types of popular feature descriptors, by using 
a large and manually-annotated MIS data set. We compared their performance based on two metrics to assess each algorithm's proficiency to detect correct correspondences, as well as the accuracy in mapping corners between images. Lowe, AMA, and Cho's exhibit competitive results, while AMA has superior performance at for the same threshold levels, because of its better adaptation to non-planar surfaces and higher robustness to outliers. The software implementations of these algorithms and our dataset are made freely available on the Internet. As future work we plan to add more types of features, and to increase the MIS dataset.

\section{References}

1. Visentini-Scarzanella, M., Mylonas, G.P., Stoyanov, D., Yang, G.-Z.: i-BRUSH: A Gaze-Contingent Virtual Paintbrush for Dense 3D Reconstruction in Robotic Assisted Surgery. In: Yang, G.-Z., Hawkes, D., Rueckert, D., Noble, A., Taylor, C. (eds.) MICCAI 2009, Part I. LNCS, vol. 5761, pp. 353-360. Springer, Heidelberg (2009)

2. Totz, J., Mountney, P., Stoyanov, D., Yang, G.-Z.: Dense Surface Reconstruction for Enhanced Navigation in MIS. In: Fichtinger, G., Martel, A., Peters, T. (eds.) MICCAI 2011, Part I. LNCS, vol. 6891, pp. 89-96. Springer, Heidelberg (2011)

3. Stoyanov, D., Darzi, A., Yang, G.Z.: Laparoscope Self-calibration for Robotic Assisted Minimally Invasive Surgery. In: Duncan, J.S., Gerig, G. (eds.) MICCAI 2005. LNCS, vol. 3750, pp. 114-121. Springer, Heidelberg (2005)

4. Hu, M., Penney, G.P., Rueckert, D., Edwards, P.J., Bello, F., Casula, R., Figl, M., Hawkes, D.J.: Non-rigid Reconstruction of the Beating Heart Surface for Minimally Invasive Cardiac Surgery. In: Yang, G.-Z., Hawkes, D., Rueckert, D., Noble, A., Taylor, C. (eds.) MICCAI 2009, Part I. LNCS, vol. 5761, pp. 34-42. Springer, Heidelberg (2009)

5. Mountney, P., Stoyanov, D., Yang, G.Z.: Three-dimensional tissue deformation recovery and tracking. IEEE Sig. Proc. Mag. 27, 14-24 (2010)

6. Su, L.M., Vagvolgyi, B.P., Agarwal, R., Reiley, C.E., Taylor, R.H., Hager, G.D.: Augmented reality during robot-assisted laparoscopic partial nephrectomy: Toward real-time 3d-ct to stereoscopic video registration. Urology 73(4), 896-900 (2009)

7. Ukimura, O., Gill, I.S.: Image-fusion, augmented reality, and predictive surgical navigation. Urol. Clin. of North America 36(2), 115-123 (2009)

8. Stoyanov, D., Mylonas, G., Deligianni, F., Darzi, A., Yang, G.Z.: Soft-Tissue Motion Tracking and Structure Estimation for Robotic Assisted MIS Procedures. In: Duncan, J.S., Gerig, G. (eds.) MICCAI 2005. LNCS, vol. 3750, pp. 139-146. Springer, Heidelberg (2005)

9. Mountney, P., Yang, G.Z.: Motion Compensated SLAM for Image Guided Surgery. In: Jiang, T., Navab, N., Pluim, J.P.W., Viergever, M.A. (eds.) MICCAI 2010, Part II. LNCS, vol. 6362, pp. 496-504. Springer, Heidelberg (2010)

10. Lowe, D.G.: Distinctive image features from scale-invariant keypoints. Int. J. Comp. Vis. 60(2), 91-110 (2004)

11. Puerto, G., Adibi, M., Cadeddu, J., Mariottini, G.: Adaptive multi-affine (AMA) feature-matching algorithm and its application to minimally-invasive surgery images. In: Proc. IEEE/RSJ Int. Conf. Intel. Rob. Syst., pp. 2371-2376 (2011) 
12. Cho, M., Lee, J., Lee, K.M.: Feature correspondence and deformable object matching via agglomerative correspondence clustering. In: Proc. 9th Int. Conf. Comp. Vis., pp. 1280-1287 (September 2009)

13. Del Bimbo, A., Franco, F., Pernici, F.: Local shape estimation from a single keypoint. In: Proc. Comp. Vis. Patt. Rec. Workshops, pp. 23-28 (June 2010)

14. Bay, H., Tuytelaars, T., Van Gool, L.: SURF: Speeded up robust features. In: Proc. European Comp Vis. Conf., pp. 404-417 (2006)

15. Mikolajczyk, K., Schmid, C.: A performance evaluation of local descriptors. IEEE Trans. Pattern Anal. 60, 1615-1630 (2005)

16. Gauglitz, S., Höllerer, T., Turk, M.: Evaluation of interest point detectors and feature descriptors for visual tracking. Int. J. Comp. Vis. 94(3), 335-360 (2011)

17. Mountney, P., Lo, B., Thiemjarus, S., Stoyanov, D., Zhong-Yang, G.: A Probabilistic Framework for Tracking Deformable Soft Tissue in Minimally Invasive Surgery. In: Ayache, N., Ourselin, S., Maeder, A. (eds.) MICCAI 2007, Part II. LNCS, vol. 4792, pp. 34-41. Springer, Heidelberg (2007)

18. Giannarou, S., Visentini-Scarzanella, M., Yang, G.Z.: Affine-invariant anisotropic detector for soft tissue tracking in minimally invasive surgery. In: Proc. 6th IEEE Int. Conf. Symp. Biom. Imag., pp. 1059-1062 (July 2009)

19. Morel, J.M., Yu, G.: ASIFT: A new framework for fully affine invariant image comparison. J. on Imag. Sciences 2(2), 438-469 (2009)

20. Hartley, R., Zisserman, A.: Multiple view geometry in computer vision. Cambridge Univ. Press (2000)

21. Fawcett, T.: An introduction to ROC analysis. Patt. Rec. Letters 27(8), 861-874 (2006) 